\title{
Концепции по обоснованию проектных решений сооружений атомных станций с учетом особых динамических воздействий
}

\author{
А.Е.Саргсян, Атомэнергопроект, Москва \\ Е.Г.Гукова, Атомэнергопроект, Москва
}

В настоящей работе изложены основные концепции по расчёту сооружений атомных станций на особые динамические природные и техногенные воздействия и обоснованию их проектных решений.

В общем объёме задач по обоснованию проектных решений сооружений атомных станций особое место отводится разработке методов расчёта сооружений при особых динамических воздействиях. В частности, повышенные требования предъявляются к сейсмостойкости и надёжности энергетических объектов и устанавливаемому на них оборудованию как при нормальном режиме эксплуатации, так и при особых динамических воздействиях.

Исследование проблемы взаимодействия сооружения с основанием с учётом геологического строения строительной площадки и конструктивных особенностей сооружения даёт возможность перейти к более обоснованным расчётным моделям.

Приведены основные актуальные направления расчёта сооружений на особые динамические воздействия. Рассмотрена специфика оценки прочности и надёжности основания, строительных конструкций сооружения, оборудования атомных станций при указанных воздействиях.

В работе изложены и систематизированы основные концепции по определению расчётных сейсмических воздействий на свободной поверхности площадки строительства атомных станций, современные методы моделирования и расчётов сооружений атомных станций, требования к определению техногенных воздействий от летающих тел и взрывов, методы расчётов защитных конструкций сооружений. Продемонстрирована практическое использование новых высокопрочных материалов, в том числе сталефибробетона, в качестве важного стратегического этапа совершенствования оптимизации компоновочных решений сооружений атомных станций. Приведена оценка эффективности применения сталефибробетона вместо обычного бетона.

Ключевые слова: атомные станции, расчёт сооружений, особые динамические воздействия, техногенные воздействия, концепции по расчёту и обоснованию проектных решений, оценка прочности, надёжность, система «основание-сооружение».

Concepts for the Justification of Design Solutions for Nuclear Power Plants Taking into Account Special Dynamic Effects

A.E.Sargsyan, Atomenergoproekt, Moscow

E.G.Gukova, Atomenergoproekt, Moscow
The basic principles of nuclear plants design analysis and justification for dynamic natural and technogenic impacts were given.

In the overall scope of the tasks of design substantiation of nuclear power plant structures, a special place was given to the development of methods for structures analysis under special dynamic impacts. In particular, increased requirements were imposed on the seismic stability and reliability of nuclear power facilities and the equipment installed on them both under normal operation and under special dynamic impacts.

Justification of necessity of taking into account the effects of interaction between the structure and the soil base presented. The study of the problem of the soil-structure interaction, taking into account the geological structure of the construction site and the structural features of the structure, makes it possible to move on to more reasonable calculation models.

The main current directions of structures analysis for special dynamic impacts are given. The specifics of assessing the strength and reliability of the foundation, structures, equipment of nuclear power plants under these impacts are considered.

The work outlines and systematizes the basic principles for determining seismic excitations on a free ground surface of the site for the nuclear plants, modern modeling methods and analysis of the nuclear plants structure, the requirements for determining technogenic impacts, and methods for calculating safety structures. Practical implementation of new high-strength materials, e.g. steel fiber-reinforced concrete that is necessary for a high-quality strategic improvement stage for layouts for nuclear power plants was demonstrated. An assessment of the effectiveness of using steel fiber concrete instead of ordinary concrete is given.

Keywords: nuclear plants, structures analysis, special dynamic impacts, technogenic impacts, concepts for design analysis and substantiation, strength assessment, reliability, soil-structure system.

В общем объёме задач по обоснованию проектных решений сооружений атомных станций особое место отводится разработке методов расчёта сооружений при особых динамических воздействиях. В частности, повышенные требования предъявляются к сейсмостойкости и надёжности энергетических объектов и устанавливаемому на них оборудованию как при нормальном режиме эксплуатации, так и при особых динамических воздействиях.

Все уникальные объекты атомной энергетики в России расположены в районах с расчётной сейсмичностью от 6 до 9 
баллов. Как правило, эти объекты возводятся на нескальных основаниях с весьма неоднородным и сложным геологическим строением, что порождает целый ряд проблем расчётного и конструктивного характера, которые практически не возникают при строительстве промышленных и гражданских объектов.

Во всех существующих теориях сейсмостойкости сооружений сейсмические воздействия на сооружения считаются заданными величинами и передаются опорным конструкциям сооружения. Предполагается, что внешнее воздействие зависит только от механизма и места расположения очага землетрясения и геологического строения строительной площадки и параметры реакций сооружения на них не влияют. При этом интенсивность сейсмического воздействия на поверхность строительной площадки в зависимости от механизма и расположения очага землетрясения чаще всего устанавливается чисто визуально. На самом же деле очевидно, что сооружение, его основание и источник первичных волн (очаг землетрясения) образуют при сейсмическом воздействии единую динамическую систему. При этом может быть существенной обратная связь: от сооружения к пластам его основания.

Расчёт сооружения в такой постановке вызывает необходимость учёта эффектов взаимодействия сооружения с основанием. В свою очередь, исследование проблемы взаимодействия сооружения с основанием с учётом геологического строения строительной площадки и конструктивных особенностей сооружения даёт возможность перейти к более обоснованным расчётным моделям.

Широкое развёртывание капитального строительства для нужд энергетики, транспорта и крупного машиностроения требует решения многих специфических задач, связанных с расчётом сооружений на динамические воздействия, в частности на воздействия ударного типа.

Особое место среди сооружений, подверженных воздействию интенсивных динамических нагрузок [повреждения осколками технологического оборудования при аварийных режимах; воздействие предметов при природных явлениях (ураган, смерч и т.д.); случайный удар падающих предметов, в частности летательных аппаратов и их частей], занимают сооружения энергетического назначения, к которым предъявляются особо высокие требования по НП-064-17¹ в отношении надёжности и безопасности при аварийных режимах и стихийных бедствиях [1].

Многие вопросы, связанные с расчётом сооружений при их соударении с летящим телом конечной жёсткости в целом, а также с расчётом их отдельных элементов, находящихся в сложном напряжённо-деформированном состоянии, на прочность при воздействии дополнительной кратковременной нагрузки большой интенсивности от локального удара летящего тела, являются актуальной тематикой в области атомной энергетики. Объекты ядерной энергетики и прежде всего атомные станции (AC) - наиболее сложные и потенциально опасные инженерные сооружения, поэтому задача обеспечения их ядерной и радиационной безопасности, в том числе при экстремальных динамических воздействиях, чрезвычайно актуальна.

Все экстремальные воздействия природного и техногенного происхождения (землетрясение, ураган, смерч, взрыв, падение самолёта и т.д.) характеризуются высокой интенсивностью, малой продолжительностью и низкой вероятностью возникновения. Поэтому оценка прочности и надёжности основания, строительных конструкций сооружения, оборудования атомных станций при указанных воздействиях представляет собой весьма специфическую проблему. Неполнота и неопределённость исходных данных по расчётным параметрам рассматриваемых воздействий и возможным сценариям повреждения и разрушения защитных конструкций сооружения, сложность реализации экспериментов на физических моделях и натурных объектах - эти обстоятельства выдвигают на первый план расчётные методы математического моделирования.

В соответствии с рекомендациями МАГАТЭ [1] установлены жёсткие требования относительно вероятности тяжёлого повреждения активной зоны ядерного реактора, которая не должна превышать $10^{-5} 1$ /год. Вероятность выхода радиоактивных веществ за пределы герметичной оболочки не должна превышать $10^{-7} 1 /$ год. Радиационное воздействие AC на население и окружающую среду не должно превышать 1 \% дозы, получаемой от источников естественной радиации.

Согласно требованиям нормативных документов РФ к проектированию атомных станций НП-031-01², MP1 5.2.05.999.0025-2011³ при обосновании сейсмостойкости элементов, относящихся к I категории ответственности, выполняющих функции по обеспечению безопасности, при выборе площадки рассматривается сейсмическое воздействие максимальной интенсивности повторяемостью один раз за 10000 лет. Воздействия от падения самолёта и воздушной ударной волны определяются из числа возможных наиболее неблагоприятных событий редкой повторяемостью 10-7 1/год по ПНАЭ Г-10-007-894, ПиН АЭ-5.65 , ПП-001-976. Расчётные параметры экстремальных нагрузок и воздействий природного происхождения (температура, снег, ураган, смерч) определяются повторяемостью один раз за 10000 лет по НП-064-17.

Правила учёта внешних воздействий природного и техногенного происхождения на объекты использования

\footnotetext{
${ }^{1}$ НП-064-17. Учёт внешних воздействий природного и техногенного происхождения на объекты использования атомной энергии / ФБУ «НТЦ ЯРБ». - Москва, 2018.

${ }^{2}$ НП-031-01. Нормы проектирования сейсмостойких атомных станций. - Москва, 2001.

${ }^{3}$ MP1.5.2.05.999.0025-2011. Расчет и проектирование сейсмостойких атомных станций. - Москва, 2011.

4 ПНАЭ Г-10-007-89. Нормы проектирования железобетонных сооружений локализующих систем безопасности атомных станций. - Москва, 1991.

${ }^{5}$ ПиН АЭ-5.6. Нормы проектирования АС с реакторами различного типа. - Москва, 1999.

${ }^{6}$ ПП-001-97. Общие положения обеспечения безопасности атомных станций. - Москва, 2001.

${ }^{7}$ НП-064-17. Учет внешних воздействий природного и техногенного происхождения на объекты использования атомной энергии / ФБУ «НТЦ ЯРБ». - Москва, 2018.
} 
атомной энергии (ОИАЭ) установлены в соответствии с ASCE STANDARD 4-98 .

В проекте ОИАЭ должны быть установлены и обоснованы значения параметров внешних природных воздействий с вероятностью возникновения в один год $10^{-4}$ и выше, а внешние техногенные воздействия определяются с вероятностью возникновения на интервале в один год и выше.

\section{Нагрузки и воздействия}

Сейсмические воздействия

Здания и сооружения, строительные конструкции и основания, технологическое оборудование атомных станций по условиям их ответственности за радиационную и ядерную безопасность подразделяются на три категории сейсмостойкости. Элементы I категории сейсмостойкости должны сохранять прочность и устойчивость при максимальном расчётном землетрясении (МР3) повторяемостью один раз в 10000 лет. Элементы II категории сейсмостойкости должны сохранять прочность и устойчивость при проектном землетрясении (ПЗ) повторяемостью один раз в 1000 лет. Элементы III категории сейсмостойкости должны сохранять прочность и устойчивость, включая землетрясение повторяемостью один раз в 100 лет. Поэтому оценку сейсмостойкости элементов III категории допускается выполнять в соответствии с общегражданским нормами по НП-031-019.

Для вновь проектируемых АС независимо от сейсмичности площадки максимальное значение горизонтальных компонент ускорения грунтов на свободной поверхности площадки при МР3 принимается по НП-031-01 равным не менее 1,0 м/ $\mathrm{c}^{2}$, а при ПЗ не менее 0,5 м/ $\mathrm{c}^{2}$.

Неопределённости, обусловленные расчётными параметрами внешних воздействий, характеристиками грунтов оснований, материалами инженерных конструкций сооружений и оборудования, учитываются:

- анализом динамического взаимодействия сооружения с основанием с учётом вариации расчётных динамических характеристик грунтов;

- расширением пиков поэтажных спектров откликов;

- использованием рекомендованных параметров демпфирования.

При строгом соблюдении указанных требований результаты расчётов будут обеспечены при $90 \%$ вероятности непревышения при $84 \%$ вероятности непревышения интенсивности исходного воздействия.

На рисунке 1 представлен график трёхкомпонентной акселерограммы колебаний грунтов на свободной поверхности площадки при МР3.

${ }^{8}$ ASCE STANDARD 4-98. Seismic Analysis of Safety Related Nuclear Structures / American Society of Civil Engineers. Approved September, 1988.

${ }^{9}$ НП-031-01. Нормы проектирования сейсмостойких атомных станций. - Москва, 2001.

${ }^{10}$ ПиН АЭ-5.6. Нормы проектирования АС с реакторами различного типа. Москва, 1999

Нагрузки на сооружения при соударении с летящим телом

В настоящее время с учётом вероятности реализации событий $10^{-6} 1$ год и выше рассматриваются различные типы летательных аппаратов. На рисунке 2 представлены силы соударения, возникающие при падении военного самолёта типа «Фантом» на сооружение атомной станции.

Учёт самолёта типа истребитель-бомбардировщик Phantom RF-4E предусмотрен рекомендациями МАГАТЭ и нормами РФ ПиН АЭ-5.6 ${ }^{10}$ [1].

Воздействие воздушной ударной волны на сооружения

Одно из основных экстремальных динамических воздействий, которое необходимо учитывать при проектировании
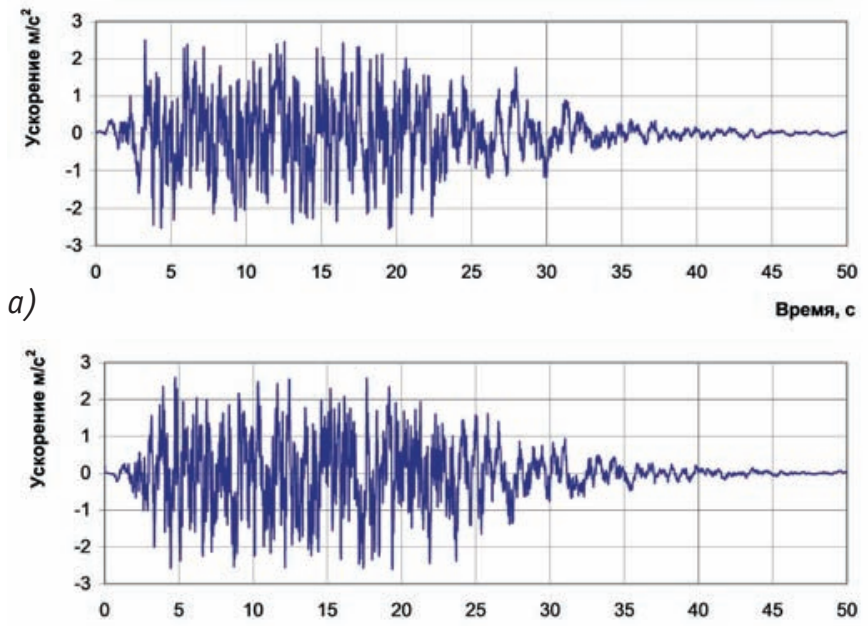

б)

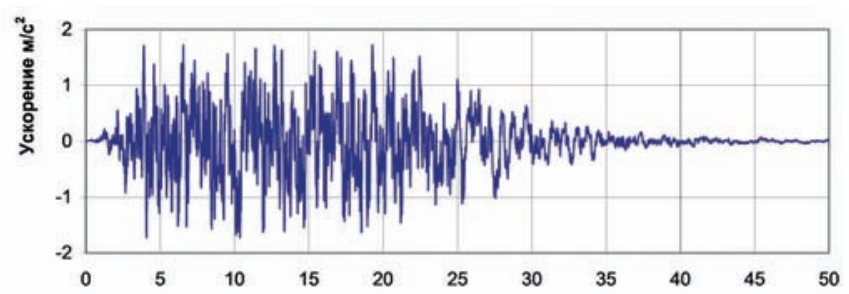

B)

Рис. 1. График трёхкомпонентной акселерограммы колебаний грунтов на свободной поверхности площадки $A C$ при MP3: а) по горизонтальной оси х (ZPA $\left.=2,5471 \mathrm{~m} / \mathrm{c}^{2}\right)$; б) по горизонтальной оси у $\left(Z P A=2,5971 \mathrm{~m} / \mathrm{c}^{2}\right) ;$ в ) по вертикальной ocu $z\left(Z P A=1,7217 \mathrm{~m} / \mathrm{c}^{2}\right)$

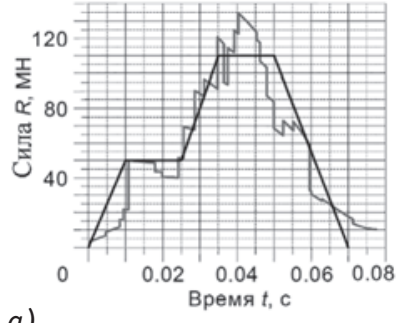

a)

Рис. 2. Силы соударения при ударе истребителя-бомбардировщика Phantom RF-4E: a) сила соударения; б) коэффициент динамичности 
a)

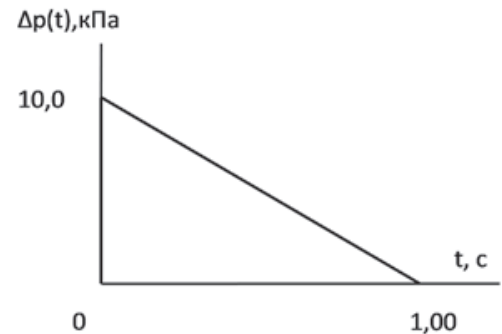

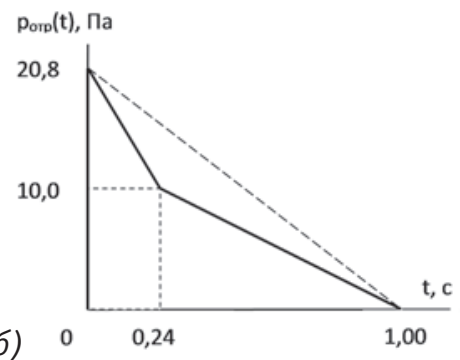

б)

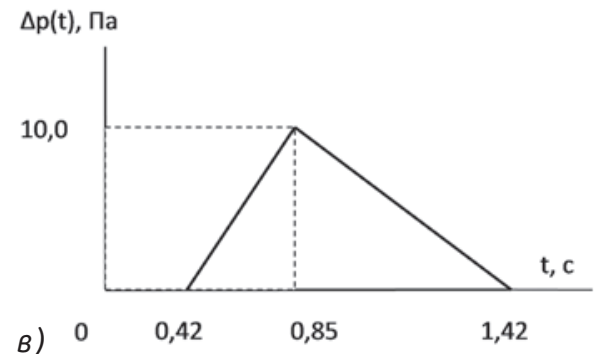

$\Delta \mathrm{p}(\mathrm{t})$, Па

в)

Рис. 3. Характер изменения давления в зависимости от времени на наружной поверхности сооружения: а) давление в проходящей волне, на кровлю и боковые стены здания; б) фронтальная стена; в) тыльная стена

сооружений атомных станций первой категории ответственности, - это воздушная ударная волна, вызванная надземным взрывом. Характер изменения давления в зависимости от времени на наружной поверхности сооружения представлен на рисунке 3 в соответствии с [1].

\section{Динамические механические модели основания сооружений}

Расчётные характеристики разработанной механической модели основания сооружений атомных станций в виде однородного инерционного полупространства представлены в таблице 1 в соответствии с рекомендациями МР1.5.2.05.999.0025-2011 ${ }^{11}$ и [1].

Интегральные жёсткости основания сооружения реакторного отделения атомной станции ВВЭР-1000 определены при заданных приведённых расчётных характеристиках грунтов основания: $\rho=2,23 \mathrm{kH} \cdot \mathrm{c}^{2} / \mathrm{M}^{4} ; \mu=0,39 ; G=739$ МПа.

\section{Методы расчёта сооружений атомных станций}

Для выполнения расчётов системы «сооружение-основание» при динамических воздействиях в настоящее время существует единственный универсальный метод.

${ }^{11}$ MP1.5.2.05.999.0025-2011. Расчёт и проектирование сейсмостойких атомных станций. Москва, 2011

Таблица 1. Квазистатические и мгновенные жёсткости основания сооружения с фундаментной конструкцией прямоугольной формы в плане [1]

\begin{tabular}{|c|c|c|}
\hline Вид движения & Квазистатическая жёсткость & Мгновенная жёсткость \\
\hline $\begin{array}{l}\text { Горизонтальное } \\
\text { поступательное } \\
\text { по оси } x\end{array}$ & $k_{x}=\frac{31, I(I-\mu) G \sqrt{L_{x} L_{y}}}{\sqrt{\pi}(7-8 \mu)}$ & $c_{,}=\frac{18,24(I-\mu) G A}{\pi(7-8 \mu)} \sqrt{\frac{\rho}{G}}$ \\
\hline $\begin{array}{l}\text { Горизонтальное } \\
\text { поступательное } \\
\text { по оси y }\end{array}$ & $k_{y}=\frac{31,1(1-\mu) G \sqrt{L_{x} L_{y}}}{\sqrt{\pi}(7-8 \mu)}$ & $c_{\gamma}=\frac{18,24(1-\mu) G A}{\pi(7-8 \mu)} \sqrt{\frac{\rho}{G}}$ \\
\hline $\begin{array}{l}\text { Вертикальное } \\
\text { поступательное } \\
\text { по оси z }\end{array}$ & $k_{t}=\frac{4 G \sqrt{L_{x} L_{y}}}{\sqrt{\pi(1-\mu)}}$ & $c_{s}=\frac{3,4 G A}{\pi \sqrt{1-\mu}} \sqrt{\frac{\rho}{G}}$ \\
\hline $\begin{array}{c}\text { Вращательное } \\
\text { относительно } \\
\text { горизонтальной } \\
\text { оси } x\end{array}$ & $k_{-}=\frac{8,52 G}{\sqrt{\pi}(I-\mu)}\left[2-\left(\frac{L_{y}}{L_{x}}\right)^{2}\right] \cdot \frac{J_{\Delta}}{\sqrt{A}}$ & $c_{\alpha}=\frac{2,1 G J_{\Delta}}{\pi(1-\mu)}\left[1+0,32\left(\frac{L_{z}}{L_{x}}\right)^{2}\right] \cdot \sqrt{\frac{\rho}{G}}$ \\
\hline $\begin{array}{c}\text { Вращательное } \\
\text { относительно } \\
\text { горизонтальной } \\
\text { оси у }\end{array}$ & $k_{e}=\frac{8,52 G}{\sqrt{\pi}(1-\mu)} \cdot \frac{J_{t}}{\sqrt{A}}$ & $c_{\sigma}=\frac{2,86 G J_{*}}{\pi(1-\mu)} \cdot \sqrt{\frac{\rho}{G}}$ \\
\hline $\begin{array}{c}\text { Вращательное } \\
\text { относительно } \\
\text { вертикальной оси Z }\end{array}$ & $k_{\mathrm{e}}=\frac{5,2 G}{\sqrt{\pi}(1-\mu)} \cdot \frac{J_{k}}{\sqrt{A}}$ & $c_{\mathrm{n}}=\frac{1,54 G J_{k}}{\pi(1-\mu)} \cdot \sqrt{\frac{\rho}{G}}$ \\
\hline
\end{tabular}

Прямое интегрирование во времени может быть использовано для расчёта как линейных, так и нелинейных систем.

Если расчётная модель системы «сооружение-основание» является линейной, то при заданных внешних динамических нагрузках интегрируется система дифференциальныхуравнений

$$
[M]\{\ddot{u}\}+[C]\{u\}+[K]\{u\}=\{P(t)\},
$$

где $[M],[C]$ и $[K]$ - матрицы масс, диссипации энергии и жёсткости соответственно;

$\{\ddot{u}\},\{u\}$ и $\{u\}-$ векторы ускорений, скоростей и перемещений, возникающих за счет собственных деформаций системы;

$\{P(t)\}=\{J\} R(t)$ - вектор внешних сил, приложенных в дискретных точках конечно-элементной модели рассматриваемой системы;

$\{J\}$ - вектор, определяющий характер распределения внешней нагрузки $R(t)$ на рассматриваемую систему с конечным числом степенями свободы.

При сейсмических воздействиях система уравнений (1) принимает вид:

$$
[M]\{\ddot{u}\}+[C]\{u\}+[K]\{u\}=-[M]\left\{J_{\mathrm{b}}\right\} \ddot{u}_{\mathrm{g}}(t),
$$

$\left\{J_{b}\right\}$ - вектор, элементы которого равны косинусам углов между направлением сейсмических колебаний основания и перемещениями по степеням свободы системы (компоненты, соответствующие угловым перемещениям, равны нулю); $\ddot{u}_{\mathrm{g}}(t)$ - закон изменения ускорений основания (акселерограммы).

Начальные условия задают перемещения и скорости перемещения по всем координатам в момент времени $t=0$ :

$\{u(0)\}=\left\{u_{0}\right\} ;\{u(0)\}=\left\{u_{0}\right\}$ (как правило, $\{u(0)\}=\{0\}$ и $\{\dot{u}(0)\}=\{0\})$.

Максимальное значение шага интегрирования по времени $\Delta t$ при применении пошаговых методов расчёта, рекомендованных различными авторами, представлено в таблице 2 в соответствии с [1].

При прямом динамическом методе пропорциональное рэлеевское демпфирование реализуется через матрицу демпфирования $[C]$, являющуюся линейной комбинацией матрицы масс $[M]$ и матрицы жёсткости $[K]$ системы

$$
[C]=\alpha[M]+\beta[K],
$$

где $\alpha$ и $\beta$ - коэффициенты пропорциональности, определяемые выражениями:

$$
\alpha=\frac{4 \cdot \pi \cdot \lambda \cdot f_{\max } \cdot f_{\min }}{f_{\max }+f_{\min }}, \quad \beta=\frac{\lambda}{\pi \cdot\left(f_{\max }+f_{\min }\right)} .
$$


Здесь $\lambda$ - значения коэффициента демпфирования; $f_{\max } n$ $f_{\min }$ - максимальная и минимальная частоты, фиксирующие частотный диапазон, в котором формируется реакция системы.

Для определения эффекта динамического взаимодействия подсистемы с общей системой необходимо воспользоваться критериями, установленными в ASCE STANDARD 4-98 12 и МР 1.5.2.05.999.0027-2011 13 .

Внешний вид конечно-элементной модели здания реакторного отделения (Р0), выполненной в программной среде (ПС) ANSYS, представлен на рисунке 4.

Расширенные обобщённые огибающие спектры отклика от MP3 на отметке $+12,200$ в зоне расположения дна бассейна выдержки при различных значениях коэффициента демпфирования в процентах от критической величины по результатам расчётов с применением ПC ANSYS представлены на рисунке 5.

\section{Оценка прочности защитных конструкций при действии локальных нагрузок, возникающих при соударении с летящими предметами}

Воздействия от падения самолёта определяются из числа возможных наиболее неблагоприятных событий редкой повторяемостью 10-7 1/год по ПНАЭ Г-10-007-8914, ПиН АЭ$5.6^{15}$, ПП-001-97 ${ }^{16}$. Расчётная схема защитной оболочки при действии локальной нагрузки [1] представлена на рисунке 6.

Использование новых высокопрочных материалов, например, сталефибробетона, является важным стратегическим этапом совершенствования оптимизации компоновочных решений сооружений атомных станций $[1,2]$.

В качестве примера в таблице 3 продемонстрирована эффективность использования сталефибробетона вместо обычного бетона в защитной оболочке РО АЭС с ВВЭР-1000.

${ }^{12}$ ASCE STANDARD 4-98. Seismic Analysis of Safety Related Nuclear Structures / American Society of Civil Engineers. Approved September, 1988.

${ }^{13}$ MP 1.5.2.05.999.0027-2011. Нормы проектирования атомных станций на сейсмостойкость. - Москва, 2011

14 ПНАЭ Г-10-007-89. Нормы проектирования железобетонных сооружений локализующих систем безопасности атомных станций. - Москва, 1991.

15 ПиН АЭ-5.6. Нормы проектирования АС с реакторами различного типа. - Москва, 1999.

${ }^{16}$ ПП-001-97. Общие положения обеспечения безопасности атомных станций. - Москва, 2001.

Таблица 2. Максимальное значение шага интегрирования по времени $\Delta \mathbf{t}$ при применении пошаговых методов расчёта, рекомендованных различными авторами

\begin{tabular}{|l|l|}
\hline Наименование метода & $\begin{array}{l}\text { Максимальное допустимое зна- } \\
\text { чение } \Delta t \text { в долях от наименьшего } \\
\text { представляющего интерес периода } \\
\text { собственных колебаний системы }\end{array}$ \\
\hline Метод Хуболта & $1 / 15$ \\
\hline Метод Ньюмарка & $1 / 10$ \\
\hline$\theta$-метод Вильсона & $1 / 10$ \\
\hline Метод Нигам-Дженингс & $1 / 5$ \\
\hline
\end{tabular}

Представленные в таблице 3 результаты расчётов показывают, что:

- при падении военного самолёта типа «Фантом», прочность оболочки, изготовленной из бетона марки В30 с толщиной $\mathrm{h}=0,6$ м, является недостаточной. Во всех других рассматриваемых случаях условие прочности оболочки выполняется с большим запасом;

- при идентичных исходных данных в случае замены обычного бетона класса В30 на высокопрочный порошковый бетон несущая способность защитной оболочки возрастает в 8,59 раза;

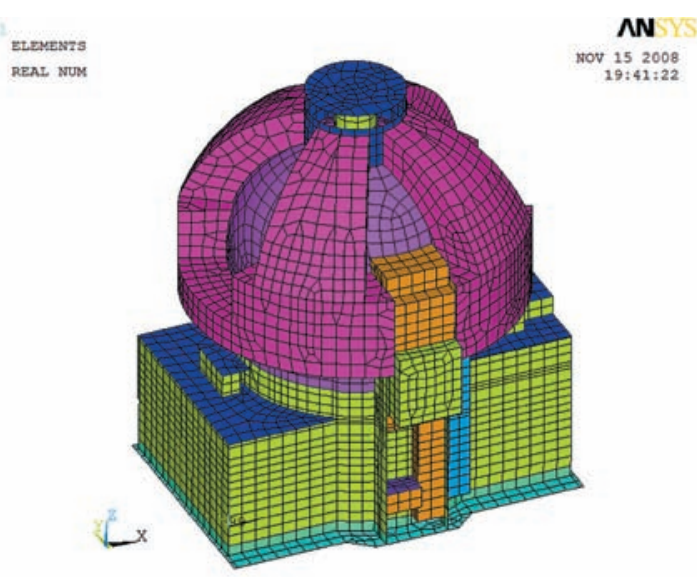

Рис. 4. Внешний вид конечно-элементной модели здания РО no ПC ANSYS

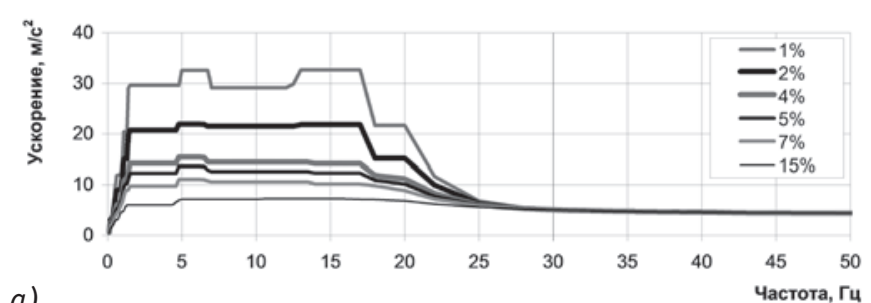

a)

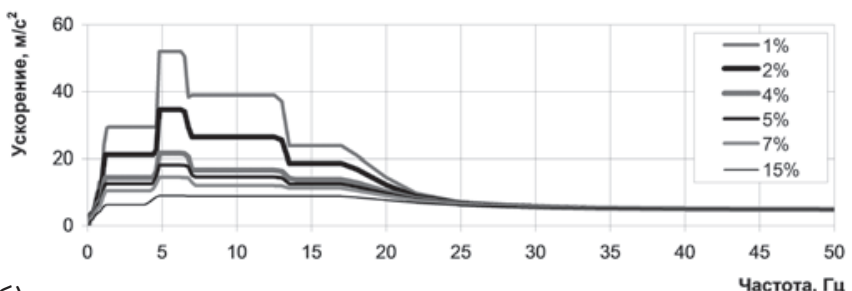

б)

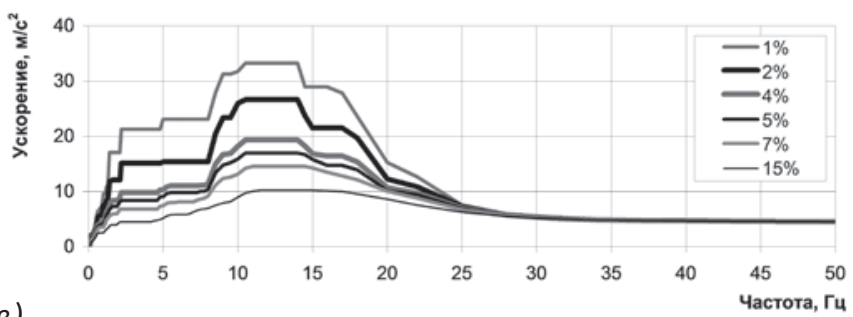

Рис. 5. Расширенные обобщённые огибающие спектры отклика от МР3 на отметке плюс 12,200 в зоне расположения дна бассейна выдержки: а) по горизонтальной оси х (ZPA = $\left.4,190 \mathrm{M} / \mathrm{c}^{2}\right)$; б) по горизонтальной оси у $\left(Z P A=4,770 \mathrm{~m} / \mathrm{c}^{2}\right)$; в) по вертикальной оси z $\left(Z P A=4,240 \mathrm{~m} / \mathrm{c}^{2}\right)$ 
- при идентичных исходных данных в случае замены обычного бетона класса В30 на сталефибробетон несущая способность защитной оболочки возрастает в 12,7 раза.

Представленные результаты расчётов подтверждают широкие возможности совершенствования конструктивных решений защитных оболочек путём использования инновационных материалов, позволяющих существенно экономить

Таблица 3. Обобщение результатов расчетов локальной прочности купольной части защитной оболочки из различных материалов при падении самолета типа «Фантом»

\begin{tabular}{|c|c|c|c|}
\hline $\begin{array}{c}\text { Толщина } \\
\text { стенки, } \\
h, \text { м }\end{array}$ & Материал & $\begin{array}{c}\text { Максимальное } \\
\text { значение силы } \\
\text { соударения, } \\
10^{5} \mathrm{kH}\end{array}$ & $\begin{array}{c}\text { Несущая } \\
\text { способ- } \\
\text { ность, } \\
P_{1 \text { max }^{\prime}} 10^{5} \mathrm{kH}\end{array}$ \\
\hline 0,6 & $\begin{array}{r}\text { Бетон марки } \\
\text { В30 }\end{array}$ & 1,1 & 0,34359 \\
\hline 1,2 & $\begin{array}{r}\text { Бетон марки } \\
\text { Взо }\end{array}$ & 1,1 & 1,75338 \\
\hline 0,6 & $\begin{array}{c}\text { Высокопроч- } \\
\text { ный порошко- } \\
\text { вый бетон }\end{array}$ & 1,1 & 2,951332 \\
\hline 0,6 & $\begin{array}{c}\text { Сталефибро- } \\
\text { бетон }\end{array}$ & 1,1 & 4,36464 \\
\hline
\end{tabular}

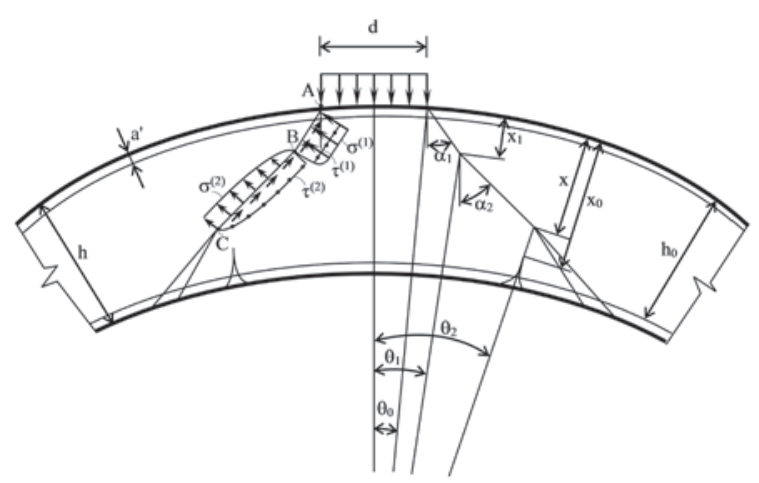

Pис. 6. Экспериментально обоснованная локальная схема разрушения железобетонной оболочки

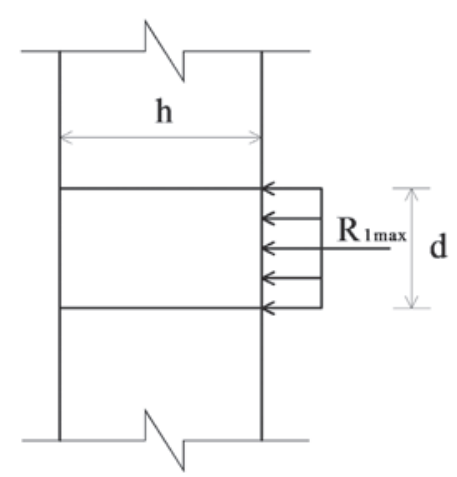

Рис. 7. Расчётная схема пробивания стеновых защитных конструкций при соударении с твёрдым телом материальные ресурсы при одновременном повышении надёжности и безопасности сооружений в атомной энергетике.

\section{Оценка прочности стеновых конструкций на пробивание при соударении с двигателем самолёта BOEING 747-400 «JUMBO JET»}

При соударении сооружения с твёрдыми телами, как правило, происходит пробивание стеновых защитных конструкций.

Расчётная схема пробивания стеновых защитных конструкций при соударении с твёрдым телом представлена на рисунке 7.

Исходные характеристики двигателя приняты в соответствии с рекомендациями компании «Арева» (AREVA):

- масса двигателя $M=1,7$ т;

- скорость двигателя в начальный момент соударения с преградой $V=100 \mathrm{~m} / \mathrm{c}$;

- площадь контакта при соударении двигателя с преградой $\mathrm{A}=1,50 \mathrm{M}$.

Диаметр пятна соударения принимает значение $d=\sqrt{\frac{4 A}{\pi}}=1,38 \mathrm{~m}$

В соответствии с рекомендациями МАГАТЭ [1] выражение силы соударения определяется по формуле $R(t)=\frac{8 M V}{m V} \sin (125 \pi t)$, где $m_{э}=1,75 T ; V_{э}=100 \mathrm{~m} / \mathrm{c}$.

Максимальное значение силы соударения в данном случае принимает значение

$$
R_{1 \max }=6860 \mathrm{kH} \text {. }
$$

Расчётное сопротивление бетона марки В50 принято $R_{\mathrm{bd}}$ $=36$ MПа. .

Выполним проверку прочности стеновой конструкции в соответствии с НП - $064-17^{17}$.

$$
R_{1 \max }=0,735 \pi d h R b d=171985 \mathrm{kH} \gg 6860 \mathrm{kH} .
$$

Следовательно, прочность стеновой конструкции на пробивание при соударении с двигателем самолёта в данном случае будет обеспечена с большим запасом.

\section{Лuтература}

1. Саргсян, A.E. Динамика и сейсмостойкость сооружений атомных станций : монография / А.Е. Саргсян. - Саров : РФЯЦВНИИЭФ, 2013. - 550 с.

2. Саргсян, А.E. Оценка прочности защитной железобетонной оболочки реакторного отделения атомной станции из бетона различных типов при падении самолёта //Academia. Архитектура и строительство. - 2012. - № 3. - С. 119-124.

3. Саргсян А.Е. Строительная механика. Механика инженерных конструкций. - М. : Высшая школа. 2008. - 462 с.

4. Sargsyan A., Elena Gukova. Evaluation of the local strength of the outer safety containment of NPP reactor building of different types of concrete to penetration due to aircraft impact / A Sargsyan., Gukova E. // SMiRT-24. - BEXCO, Busan, Korea, 2017, August 20-25.

17 НП-064-17. Учет внешних воздействий природного и техногенного происхождения на объекты использования атомной энергии / ФБУ «НТЦ ЯРБ. - Москва, 2018. 
5. Бирбраер, А.Н. Экстремальные воздействия на сооружения / А.Н. Бирбраер, А.Ю. Роледер. - СПБ, Издательство Политехнического университета, 2009.

6. Birbraer A.N. Probabilistic Assessment of NPP Building Structures Strength under Aircraft Impact. Paper No. 1644 / A.N. Birbraer, A.J. Roleder, S.B. Arhipov // Trans. 16th International Conference on Structural Mechanics in Reactor Technology (SMiRT 16), Washington DC, Aug. 2001.

7. Riera J.D. A Critical Reappraisal of Nuclear Power Plant Safety Against Accidental Aircraft Impact / J.D. Riera // Nucl. Engng. and Des. - 1980. - Vol. 57. - P. 193-206.

8. Zorn N.F. On the Failure Probability of the Containment under Accidental Aircraft Impact / N.F. Zorn, G.J. Schuëller // Nucl. Engng. and Des. - 1986. - Vol. 91. - P. 277-286.

9. Sargsyan A. Development and Substantation of Dynamic Mechanical Model of NPP Structures Foundation Represented aslnertia Homogeneous Halfspace in 3D Problem Definition / A. Sargsyan, E. Gukova, A. Grishin // International Conference SMiRT 22. - San Francisco, USA. 2013. - P. 53-64.

10. Sargsian A. Substantiation of Reliability of Developed Spatial Static and Dynamic Models of Natural and Pile Foundation of NPP Structures / A. Sargsian, E. Gukova, V. Geraschenko // SMiRT-23.- Manchester, United Kingdom, 2015, August 10-14, 2015.

11. Sargsyan A., Grishin A., Gukova E. Generation of dynamic mechanical model of inertial soil foundation in consideration of NPP structures basement finite stiffness / A. Sargsyan, A. Grishin, E. Gukova // International Conference SMiRT 20. Helsinki, Finland, August 9-14, 2009.

\section{References}

1. Sargsyan A.E. Dinamika i seismostoikost' sooruzhenii atomnykh stantsii [Dynamic and Seismic Stability of the NPP Structures] monografiya. Sarov, RFYaTs-VNIIEF Publ., 2013, 550 p.

2. Sargsyan, A.E. Otsenka prochnosti zashchitnoi zhelezobetonnoi obolochki reaktornogo otdeleniya atomnoi stantsii iz betona razlichnykh tipov pri padenii samoleta [Evaluation of the strength of t outer safety containment of NPP reactor building of different types of concrete to penetration due to aircraft impact]. Academia. Arkhitektura i stroitel'stvo [Academia. Architecture and construction], 2012, no. 3, pp. 119-124.

3. Sargsyan A.E. Stroitelnaia Mekhanika. Mekhanika In zhenernykh Konstrukciy [Structural Mechanics. Mechanics of Engineering Constructions]. Moscow, Vyshaia Shkola Publ., 2008.

4. Akop Sargsyan, Elena Gukova. Evaluation of the local strength of the outer safety containment of NPP reactor building of different types of concrete to penetration due to aircraft impact. SMiRT-24. BEXCO, Busan, Korea, August 20-25, 2017.

5. Birbraer A.N., Roleder A.Y. Ekstremal'nie vozdeistviya na sooruzhenia [Extreme Impacts on the Structures]. SPB, Izdatelstvo Politekhnicheskogo universiteta Publ., 2009

6. BirbraerA. N., RolederA. J., ArhipovS. B. Probabilistic Assessment of NPP Building Structures Strength under Aircraft Impact. Paper No. 1644. Trans. 16th International Conference on Structural Mechanics in Reactor Technology (SMiRT 16). Washington DC, Aug. 2001.

7. Riera J. D. A Critical Reappraisal of Nuclear Power Plant Safety Against Accidental Aircraft Impact. Nucl. Engng. and Des., 1980, Vol. 57, pp. 193-206.

8. Zorn N. F., Schuëller G. J. On the Failure Probability of the Containment under Accidental Aircraft Impact. Nucl. Engng. and Des., 1986, Vol. 91, pp. 277-286.

9. Sargsian A., Gukova E., Geraschenko V. Substantiation of Reliability of Developed Spatial Static and Dynamic Models of Natural and Pile Foundation of NPP Structures. SMiRT-23. Manchester, United Kingdom, 2015, August 10-14.

10. Sargsyan A., Gukova E., Grishin A. Development and Substantation of Dynamic Mechanical Model of NPP Structures Foundation Represented aslnertia Homogeneous Halfspace in 3D Problem Definition. International Conference SMiRT 22. San Francisco, USA, 2013, pp. 53-64.

11. Sargsyan A., Grishin A., Gukova E. Generation of dynamic mechanical model of inertial soil foundation in consideration of NPP structures basement finite stiffness. International Conference SMiRT 20. Helsinki, Finland, 2009, August 9-14.

Саргсян Акоп Егишович (Москва). Доктор технических наук, профессор, академик МАИ при 00Н. Начальник Научно-исследовательского отдела динамики и сейсмостойкости А0 «Атомэнергопроект» (107996, Москва, Бакунинская ул., д. 7, стр. 1. Атомэнергопроект).

Гукова Елена Геннадиевна (Москва). Главный специалист А0 «Атомэнергопроект» (107996, Москва, Бакунинская ул., д. 7, стр. 1. Атомэнергопроект). Эл. почта: gukova_eg@aep.ru.

Sargsyan Akop Egishovich (Moscow). Doctor of Technical Sciences, Professor, Academician of the Moscow Aviation Institute at the UN. Head of the Research Department of Dynamics and Seismic Resistance of AO "Atomenergoproekt" (7 Bakuninskaya st., b. 1, Moscow, 107996. Atomenergoproekt).

Gukova Elena Gennadievna (Moscow). Chief Specialist of Atomenergoproekt A0 "Atomenergoproekt" (7 Bakuninskaya st., b. 1, Moscow, 107996. Atomenergoproekt).E-mail: gukova_eg@aep.ru. 